\title{
Impact on facial rejuvenation with dermatological preparations
}

\author{
Patrick J Bowler \\ Court House Clinics, London, UK
}

Correspondence: Patrick J Bowler

Court House Clinics, 30 B

Wimpole St, London, UK

Tel +448455555050

Email pbowler@courthouseclinics.com

\begin{abstract}
The treatment options for facial rejuvenation using dermatological, nonsurgical techniques have dramatically increased in the past 10 years. This follows the introduction of botulinum toxin and a variety of dermal fillers. The public interest in noninvasive treatments has changed the market beyond recognition with more physicians involved in providing services to satiate the demand. The impact on the public and medical profession is discussed.
\end{abstract}

Keywords: facial aging, botulinum toxin, dermal fillers, combination therapy, cosmetic market

\section{Introduction}

\section{Definition of facial rejuvenation}

This review is concerned with the concept of facial rejuvenation utilizing dermatological preparations in a nonsurgical approach. The combination of art and science now provides physicians with the capability to make the face appear more youthful. Facial aging combines intrinsic or genetically determined and extrinsic factors including sun exposure, smoking, diet, and general lifestyle. The features of the aging face are well described and include loss of skin elasticity, lines, wrinkles and dyspigmentation. The management options are focused on moderating and even reversing some of these changes

\section{The market}

There are few reliable statistics in the UK. A Mintel ${ }^{\mathrm{TM}}$ report $^{1}$ stated the number of cosmetic surgery operations in the UK in value terms had increased from $£ 450$ million in 2005 to $£ 900$ million in 2007, a 100\% increase over three years. In 2005, this included 230,000 nonsurgical procedures such as Botox ${ }^{\mathrm{TM}}$ and fillers which rose to 472,000 in 2007. In the UK, the British Association of Aesthetic Plastic Surgeons (BAAPS) ${ }^{2}$ produces reliable cosmetic surgery figures but they only relate to its members. It is not known what percentage they form of the total number of UK procedures. In 2007 , they performed 32,453 procedures, $12.2 \%$ up on 2006 .

In the US, there are more reliable indicators produced by surgical audits from the American Society for Aesthetic Plastic Surgery (ASAPS) (Figure 1).

There were almost 11.7 million cosmetic procedures in the US in 2007 , of which $18 \%$ were surgical and 82\% nonsurgical. From 1997 to 2007, surgical procedures increased by $114 \%$ and nonsurgical by $754 \%$. However in the year 2006-2007 there was only a 2\% overall increase. The dramatic increases occurred between 1997 and 2004 with a leveling off in the past four years (Figure 2). Women account for $91 \%$ of procedures, but significantly men had a year of increase of $17 \%$ from 2006 . In nonsurgical care, the main procedures were: Botox ${ }^{\mathrm{TM}} 23 \%$, dermal fillers $18 \%$, and laser hair removal $14.6 \%$.

\section{Influences in the market}

The main forces in the market place are the written media, TV shows, radio, and celebrity endorsement and peer pressure. There are many sociological and psychological factors 


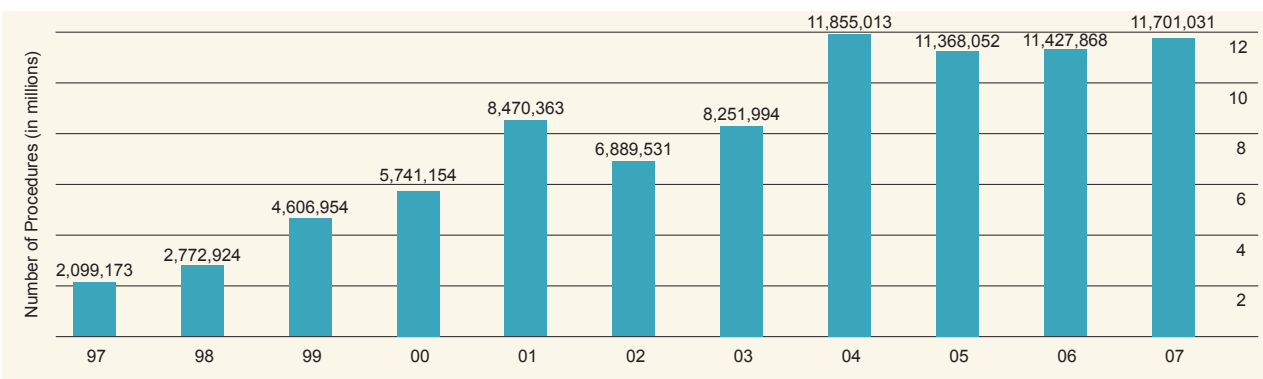

Figure I Cosmetic surgery (surgical and nonsurgical cosmetic procedures: totals). Copyright @ 2008. American Society for Aesthetic Plastic Surgery.

that influence individual choices, which are beyond the remit of this review. Magazines often include features on cosmetic work with prominence given to "celebrities" which may have an influence on public opinion. In the UK, television series such as "10 Years Younger" and "Extreme Makeover" have brought into the comfort of our homes vivid illustrations of how the worst possible cases can be transformed. Women and men are under pressure to appear youthful and healthy. High separation and divorce rates, peers, work and career prospects all have a significant influence. The increased public awareness and the Internet are probably the most important features driving the market forwards.

\section{Demand for nonsurgical care}

We are at present in the middle of a minimally invasive, no downtime, socially convenient, quick recovery, costconscious revolution. The public is seeking easy, pain-free methods of appearing youthful and healthy without the problems of invasive cosmetic surgery. This demand has had significant impact on:

1. Suppliers of dermatological preparations and other noninvasive technology. The pressure to produce new innovative products is high which can lead to premature launches

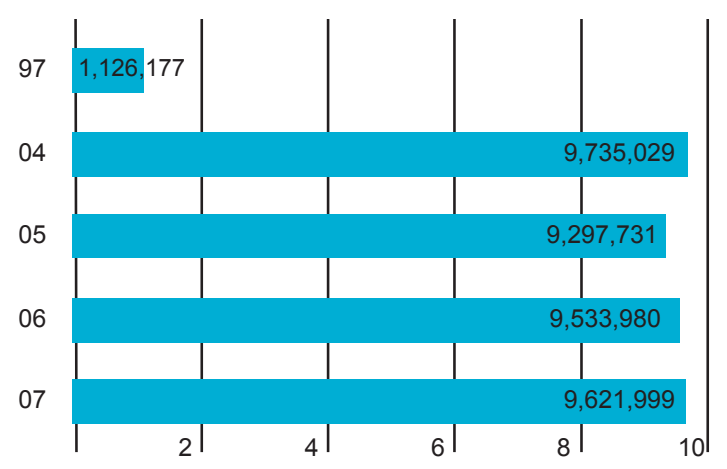

Figure 2 Nonsurgical cosmetic procedures (millions). Copyright $\odot$ 2008. American Society for Aesthetic Plastic Surgery. before there is an opportunity for adequate clinical assessment. Unfortunately in the EU the regulations relating to the introduction of fillers are not demanding. This has led to over 70 filler products being currently available which causes professional and public confusion. At this time in the US where there is a more vigorous regulatory approach, there are less then 10 approved fillers. There are some pharmaceutical companies involved, Allergan (Irvine, CA), the manufacturer of Botox ${ }^{\mathrm{TM}}$ being a major player. They have recently added fillers, skincare and breast implants to their portfolio. Others are sure to follow.

2. For the medical profession in the UK, there is now pressure to find high standard, independently validated training courses. The Royal Colleges in the UK have to recognize the public and professional interest and become more actively involved in education and training.

3. Governments need to understand the importance of regulation in order to elevate standards and ensure public safety.

\section{Differentiation of nonsurgical/surgical care}

Up to the 1980s, the famous and privileged were the only segments of the population considering cosmetic enhancement. The procedures were limited to surgery and deep acid peels. The introduction of wrinkle fillers and botulinum toxins heralded the onset of the nonsurgical era, providing low risk, effective but subtle enhancements.

\section{Present nonsurgical options}

\section{Skin care at home}

Effective skin care is one of the most neglected areas in cosmetic medical practice. Although Doctors have been convinced of the benefits of sunscreens and moisturisers, the concept of skin rejuvenation using creams and lotions is viewed with a high degree of scepticism. In the 1980s, Dr Albert Kligman ${ }^{3}$ induced a radical change of 
thought by demonstrating that sun-damaged skin could be rejuvenated using trans-retinoic acid, a vitamin $\mathrm{A}$ derivative. This led to Retin $\mathrm{A}^{\mathrm{TM}}$ being given approval, as a topical, prescription only agent, for the treatment of sun-damaged skin. This single event triggered incredible interest and investment from cosmetic manufacturers and pharmaceutical companies determined to be involved in a hugely competitive and profitable business. Now, in 2008 medical practice and our patients have benefited from this research and development. We have a number of tools and options to help improve skin health and even reverse the adverse effects of UV light.

Skincare should be viewed as an essential part of cosmetic practice. We know we can efface lines using injectables, but if this is put against the general background of skin that is smooth, moist, evenly pigmented and radiant, the overall improvement can be dramatic. For optimum results every patient should be using medically approved skincare, recommended by the doctor as part of a homecare program.

\section{Essentials of skincare Indications}

The main indications for medical skincare:

- Dryness

- Loss of elasticity

- Hyperpigmentation

- Fine lines

- Oily, acne prone.

\section{Management of skincare Lifestyle advice}

We are aware patients do not want to hear advice on changing lifestyle with its consequently low chance of compliance. It is imperative time is taken in the consultation process to ensure they understand these changes are an important part of a skin care program and that is their responsibility. The doctor can make recommendations but not supervise daily applications of topical agents. The important factors and advice that need to be addressed include: sun exposure, tan bed usage, smoking, stress, diet, and lifestyle issues.

\section{Product ingredients}

The number of ingredients in skin products is bemusing to doctors yet alone our patients. Keeping it simple is best for all concerned. There are three main groups: cosmetics, cosmeceuticals, and pharmaceuticals. By definition cosmetics are not, by law, able to alter the structure or function of the skin, so are basically limited to moisturization effects.
The term cosmeceuticals was first used by Albert Kligman in the 1980s and comprises a growing group of agents mid way between cosmetics and drugs. The companies are reluctant to enter the extremely expensive domain of drug development and regulatory approval. So they are content to continue promoting "cosmeceutical" products that they claim work but are not up to drug-approved status.

To date retinoids are the only prescription-only medicines (POMs) available in topical products.

\section{Alpha hydroxy acids}

Alpha hydroxy acids (AHAs) are exfoliators and moisturizers commonly found in many different concentrations in skincare products. Alpha hydroxy is the name for a group of acids that are derived from foods, such as glycolic, lactic, maleic and tartaric acids, which decrease stratum corneum thickness by reducing corneocyte adhesion at differing levels. ${ }^{4}$ Histologically Ditre and colleagues showed that AHAs increase collagen density and mucopolysacccharides, causing increase in dermal thickening. ${ }^{5}$ Kligman demonstrated, by combining glycolic acid $8 \%$ and tretinoin $0.1 \%$, a significant improvement in wrinkles and skin smoothness, suggesting there may be some synergy between the two ingredients. ${ }^{6}$ Medical AHA products are of higher concentration and acidity than over-the-counter (OTC) products. A test patch is advisable to exclude any sensitivities.

\section{Retinoids}

There is overwhelming evidence supporting the efficacy and safety of topically applied retinoids in the treatment of photodamaged skin. Tretinoin was approved as the first topical POM in the treatment of photodamaged skin in the $1990 \mathrm{~s}^{3}$ Epidermal effects include normalization of keratinocyte life cycles, decreased keratinocyte atypia and normalization of melanosome dispersion. Dermal changes show increased collagen, elastin, and glycosaminoglycan (GAGs) deposition. Visible evidence of photodamage can be significantly improved. From a histological viewpoint structural changes in solar elastosis and collagen degeneration are demonstrated with restoration of Langerhans cells and correction of dysplastic changes. ${ }^{7}$ Tazarotene, a retinoid analogue, has displayed similar effects to tretinoin. ${ }^{8}$

Patients must be thoroughly educated on the correct use of retinoids. A gradual increase in frequency of application is advised to enable tolerance to establish. Apply at night, twice weekly to start, increasing to every night over 4-6 weeks. They must expect mild redness and scaling but this subsides with use of additional moisturizer. The maximum effect may 
take 12 months following which a maintenance regime of three times per week can be introduced. Retinoids should not be used in pregnancy. Clients will be more sun sensitive so concurrent use of sun protection is mandatory.

\section{Vitamin C}

Topical Vitamin C is commonly incorporated into skin products because of its stimulatory effect on collagen synthesis, its ability to regenerate vitamin $\mathrm{E}$ and an antioxidant effect. ${ }^{9}$ It has also been found to inhibit tyrosinase and so reduce areas of hyperpigmentation. There is some protection from UV radiation and improvement in some inflammatory skin conditions due to its antioxidant effects. ${ }^{10}$ The active form of vitamin C, L-ascorbic acid is unstable so more stable esterified forms have been formulated. Ascorbyl palmitate and magnesium ascorbyl phosphate are common ingredients in skin care products that convert to the active form on skin contact. Various studies have shown some improvement in photodamage. ${ }^{11}$

\section{Vitamin E}

Vitamin E encompasses a family of substances with antioxidant and moisturizing activity. Preclinical studies showed topical vitamin E protects against UVB-mediated damage. It is commonly incorporated into cosmetic and cosmeceutical preparations. ${ }^{12}$

\section{Idebenone}

Derived from coenzyme q10, idebenone is a powerful antioxidant and is believed to stimulate the production of collagen. McDaniel demonstrated it to be superior in a comparative study to other antioxidants ${ }^{13}$ and its clinical effectiveness in treating photodamaged skin. ${ }^{14}$ It is effective in reducing wrinkles and improving the overall condition of the skin after prolonged use.

\section{Moisturizers}

In medical cosmetic practice, we must recommend products that have proven ability to improve damaged skin. Moisturizers on their own have limited use in skin rejuvenation and there is huge competition from the OTC market. A moisturizing sunscreen has a double benefit and raises compliance levels.

\section{Hydroquinone}

Hydroquinone is a POM in the UK, but is banned OTC in Europe because of exogenous ochronosis, a severe hyperpigmenting damaging side effect. It is, however, the most effective depigmenting product available that inhibits tyrosine production. Recommended use is for 4-6 week periods every 12 weeks, response-dependent. Azelaic acid and kojic acid are alternatives. ${ }^{15}$ Kligman developed a formula containing hydroquinone, retinoic acid and prednisone which is useful in difficult cases but very unstable with a short shelf life of under four weeks.

\section{Hormone replacement therapy}

It is well documented that estrogen loss has a profound effect on the skin, which becomes thinner and less hydrated. In suitable patients, hormone replacement therapy has been repeatedly demonstrated to rehydrate, increase collagen content, elasticity and dermal thickness. ${ }^{16}$

\section{Peptides}

I mention this group of cosmeceutical agents briefly because there is high public awareness due to promotion of their products by cosmetic companies. In particular, there are claims that these topical agents can produce botulinum toxin-like effects. There is no significant evidence at this point that they work as anything other than moisturizers. However, they compose an interesting group which may have a place in the future.

\section{Sunscreens}

Sunscreens are essential for skin health and rejuvenation. Recent advances in formulations have made products easier to apply and more cosmetically appealing. Sunlight penetrating the Earth's atmosphere exposes us to UVA and UVB ranging from 290-400 $\mathrm{nm}$. Sunscreens are labeled with an sun protection factor (SPF) number which is only a measure of UVB protection. UVA has a longer wavelength than UVB and goes deeper into the skin, causing damage in the dermis. At this time there is no universally agreed method of labeling for UVA protection. Most products now contain two types of ingredients: (a) reflective agents, for example, zinc oxide or titanium dioxide. They are inert, unlikely to cause any allergic reaction and simply reflect the UV light; and (b). Chemicals that absorb and react with sunlight to render it harmless. Common ingredients are avobenzone, oxybenzone, and octyl methoxycinnamate. These cover most of the wavelengths but no sunscreen provides $100 \%$ protection. In the future, increasingly powerful antioxidants will be added into products so that, in theory, damage is repaired as it arises. To encourage compliance, moisturizers combined with SPF are usually recommended. Daily application is required and not only to the face but neck, hands, and décolleté. Men should be reminded to cover their bare heads and tops of ears. 


\section{Skin rejuvenation treatments in clinic Chemical peels}

Chemical peels have been used for many years in skin rejuvenation. All peels are derived from basic chemicals known to inflame, exfoliate, and destroy skin cells in a controlled manner. These effects can improve sun damage, solar lentigines, wrinkles, acne, melasma, and scarring. By peeling off the damaged cells, new ones come to the surface and produces a smoother, healthier-looking skin. Deeper peels can also induce new dermal collagen formation which can improve fine lines and wrinkles. It is essential to maintain the benefits by using medical skincare and sun protection throughout the year. ${ }^{17,18}$

It is easy to be confused by trade names given to peeling agents, but a simple classification is based on their depth of penetration into the skin.

- Superficial peels: glycolic acid, salicylic acid and Jessners solution, 10\% trichloroacetic acid (TCA). These peels are used to treat mild sun damage, acne and melasma. They freshen up the skin, giving a healthy glow. A course of 6-8 is usually recommended with maintenance sessions every three months. Do not expect noticeable changes with this type of peel, but it is safe with very limited side effects.

- Medium peels: Strong Jessners solution, Resorcinol, 15\%-20\% TCA, low concentration phenol. This depth of peel is for fine lines, wrinkles, mild photodamage, superficial scars and pigmentation. The skin does exfoliate and there is a recovery period of several days. Side effects include redness and occasionally increased pigmentation, especially in darker skin types.

- Deep peels: Phenol, 30\% TCA. Not so frequently used today these deep peels have mostly been replaced by lasers. They are used to treat deeper lines and advanced sun damage. There is a $2-3$ week recovery time and again persisting redness, infection, increased pigmentation and scarring are possible side effects.

Chemical peels are an important tool in the treatment of a variety of skin conditions. It is essential that good homecare products and adequate skin protection be used before and after peels to obtain the best and long lasting results. They can be extremely effective in their own right as well as enhancing the benefits of other treatments such as botulinum toxin, fillers, and cosmetic surgery.

\section{Microdermabrasion}

Microdermabrasion used to be the preserve of aestheticians, but systems for doctors have been introduced that allow any depth in the skin to be reached. The general consensus is that microdermabrasion is a superficial treatment capable of enhancing the skin's appearance, but does not show any significant effect on wrinkles. There is very limited clinical data on microdermabrasion. ${ }^{19}$

\section{LED light}

The use of red and infrared nonlaser light has been shown to have beneficial effects in skin rejuvenation, not by thermal damage but through photobiomodulative reactions. The various tissue and cell types in the skin have their own absorption characteristics, absorbing light at specific wavelengths. Studies have demonstrated that selective wavelengths have stimulatory effects on cell types. Most importantly this has been demonstrated in the formation of collagen precursors and enzymes associated with cellular development. This technology offers a means of delivering light to treat large surface areas with no significant adverse effects. A detailed study by Lee and colleagues showed significant wrinkle reduction, up to a maximum of $36 \%$ and improvement in skin tone. ${ }^{20}$

This technology is undoubtedly an important advance in skin rejuvenation. There has been particular interest by some physicians when used as the light activating source in photodynamic light therapy using topical drugs such as 5-fluorouracil and 5 aminolevulinic acid. More investigation is underway to verify results and determine optimal treatment regimes. ${ }^{21}$

\section{Lasers and intense pulse light}

The first technologies for skin rejuvenation began in the early 1990s with the use of carbon dioxide lasers. These are powerful machines that burn off the epidermis and down into the reticular dermis. This enabled the skin to reform and appear youthful and regenerated. In the dermis, significant changes occurred with remodeling of collagen fibres, which developed up to twelve months following the treatment. However, the effectiveness of the technique was limited by discomfort during and after the procedure, a 3-4 week recovery period, redness and hyper- and hypopigmentation. The latter can appear 1-2 years post-treatment, with no effective treatment. Erbium-Yag lasers were then introduced which were marketed with reduced healing time with lower complication rates. However, this improvement proved to be minimal. All this led to a reduced demand for so called "ablative" laser resurfacing. A place remains for this treatment, for example, in severely photodamaged or scarred patients, but should only be performed in specialist centers by experienced physicians and surgeons. 


\section{Fractional lasers}

Fractional lasers treat a fraction of the skin at a time. Using tried and tested $\mathrm{CO}_{2}$ and Erbium-Yag and other lasers, the energy is delivered in fractionated columns with up to $30 \%$ of the skin being ablated with a single pulse. This leads to quicker recovery times but multiple treatment sessions are necessary. There are no studies yet showing how effective these lasers are compared to existing technology. It is not yet known if the trade-off of rapid recovery gives sufficiently good results.

The demand for new procedures that provide optimal results with minimal side effects has continued to rise. As with many advances, another came serendipitously. Patients having hair removal with Nd-Yag lasers and later intense pulsed light (IPL) systems noticed their skin texture and tone improved. Histology showed some remodeling of collagen in the upper dermis, but not to the degree of $\mathrm{CO}_{2}$ lasers. However, now there are many systems on the market claiming effects for "nonablative lasers and IPLs." It is generally agreed that these machines produce subtle changes not always visible on photographs but with some degree of patient satisfaction. Claims for significant wrinkle reduction have to be treated with scepticism. ${ }^{22}$

\section{Radio frequency infrared technology for facial skin tightening}

Radio frequency infrared technology for facial skin tightening is as yet an unproven technology with some anecdotal evidence for producing a degree of skin tightening by dermal heating and inducing collagen contraction. This can be helpful in reducing the appearance of jowls and nasolabial folds. ${ }^{23}$

\section{Laser hair removal}

Undoubtedly excessive facial hair can be socially isolating. There are many peer reviewed articles attesting to the effectiveness of laser/IPL hair removal systems. Preston and Lanigan reported high patient satisfaction rates. ${ }^{24}$ A psychological benefit can arise, which in itself improves facial appearance.

\section{Minor surgery: Removal of lumps and bumps}

Simple excision of cosmetically unacceptable lumps and bumps can have a surprising effect on the skin and contributes to the look good, feel good factor.

\section{Dermal fillers}

Silicone injections were the early dermal fillers but problems relating to permanency, granulomas and spread from site of injection led to its downfall. In the early 1980s, the first temporary wrinkle filler using bovine collagen became accepted by physicians and the public. It was an innovative product but had the downside of a 3\% allergic reaction rate. Scientists in the EU were researching hyaluronic acid (HA), a glycosaminoglycan which is strongly hydroscopic, hydrates, and adds volume to the skin. HA fillers rapidly became the most popular product lines and gained Food and Drug Administration (FDA) approval in 2003. There have been attempts at introducing human-derived dermal fillers, but without significant success. More recently, products that add volume and last longer have become an important part of aesthetic dermatological practice, the most established being poly-L-lactic acid (PLLA). HA formulations and calcium hydroxyl appetite products are newer alternatives. Fillers maybe divided into categories; temporary, semipermanent, and permanent, line fillers and volume enhancers. The agreed best practice is the use of temporary HA fillers as line fillers and PLLA or HA as volume enhancers.

\section{Hyaluronic acid fillers}

The high allergy rate of bovine collagen provided a market entry for HA fillers because of their significantly reduced allergic response. HA is identical in all species, leading to high acceptability in humans. ${ }^{25}$ In addition it has been shown to have a longer duration. ${ }^{26} \mathrm{HA}$ fillers can last 6-12 months and in some clients the author has seen 12-24 months of clinical effect. This unexpectedly long duration of activity maybe partially explained by Wang who demonstrated that cross linked HA (Restylane ${ }^{\mathrm{TM}}$ ) stimulates collagen synthesis. ${ }^{27}$ There are many HA products on the market, but the main providers are Q-Med (Uppsala, Sweden) and Allergan. The pros and cons of these various products are small and related to HA concentration and cross linking. Allergan have recently introduced an HA product containing lidocaine which is gaining popularity with patients. Pinsky and colleagues showed Juvederm ${ }^{\mathrm{TM}}$ to be safe and effective. ${ }^{28}$

No skin tests are necessary and treatment can, in some circumstances, be given at the first consultation. They are injected into the mid dermis to fill nasolabial folds, marionette lines, chin and jowl depressions, depressed scars, lip lines, redefining lip edge, and adding lip volume.

Common side effects include erythema, redness, and bruising. These are short lived and resolve spontaneously after up to seven days. Poor injection technique, usually placing the material too superficially, results in a bluish tinge and small nodules which can be long-lasting. This can be corrected by using hyaluronidase, an enzyme that 
degrades HA. Delayed hypersensitivity with granulomas can occur in any HA products but is rare. Specific treatment protocols are recommended using local steroid injections and anti-inflammatory agents. ${ }^{29}$

\section{Volume enhancers}

Poly-L-lactic acid (PLLA) is a synthetic, biodegradable inert peptide polymer originally used in treating facial lipoatrophy induced by HIV drug therapy. ${ }^{30}$ The potential in cosmetic use quickly became obvious, opening new possibilities in the nonsurgical approach to facial rejuvenation. Redefining malar prominences, softening nasolabial folds and replacing loss of volume in the cheeks has the potential to dramatically alter facial appearance. It is thought to stimulate fibroblasts laying down new collagen. There is therefore a gradual increase of volume. ${ }^{31}$ Four to six sessions are usually required with maximum benefit taking up to six months. Effects last 18-24 months. PLLA has a good safety record. Evolving protocols have reduced the incidence of granulomas and nodule formation. ${ }^{32}$ The use of PLLA is technique-dependent and results maybe variable depending on patient selection and physician experience.

Q-Med and Allergan have introduced high concentration HA products, Sub-Q $\mathrm{Q}^{\mathrm{TM}}$ and Voluma ${ }^{\mathrm{TM}}$, to add facial volume. They have the advantage of generally only requiring one treatment and easy to use but the disadvantage of lasting approximately 12 months.

Volume enhancers have been a significant nonsurgical advance in the treating the aging face. It is now possible to alter facial shape to attain a more youthful appearance. Public awareness is still relatively low compared to wrinkle fillers but this will inevitably change.

\section{Botulinum toxins}

Botulinum toxins and Botox ${ }^{\mathrm{TM}}$ in particular revolutionized the nonsurgical cosmetic market since its introduction in late 1980s. Cosmetic use of botulinum toxin began when Carruthers and Carruthers coincidentally noted an improvement in periorbital appearance in patients undergoing treatment with botulinum toxin type-A for blepharospasm. Initial cosmetic indication was the glabellar area, but its use has now expanded to successfully treat a variety of hyperfunctional facial lines, such as peri orbital wrinkles (crow's feet), lower eyelid wrinkles, nasolabial lines and horizontal forehead rhytides. ${ }^{33-35}$ Today, its use for treatment of hyperfunctional facial lines has become the most popular cosmetic procedure, ${ }^{36}$ with high patient satisfaction rates. ${ }^{37}$

Botox $^{\mathrm{TM}}$ was licensed for cosmetic use in the US in April 2002 and March 2006 in the UK. There are numerous studies confirming its efficacy and safety. Dysport ${ }^{\mathrm{TM}}$ is an alternative Botulinum type A but with slightly different characteristics to Botox ${ }^{\mathrm{TM}}$ in terms of side effects and clinical benefit. The most important difference is that Dysport's ${ }^{\mathrm{TM}}$ effect spreads further from the site of injection. ${ }^{38}$ This can be particularly useful in the periorbital area but a disadvantage in the forehead where brow ptsois may be a problem. Combining the two products can be a positive way forward. A Belgian study ${ }^{39}$ suggested clients previously treated with Botox $^{\mathrm{TM}}$ are less satisfied than with Dysport ${ }^{\mathrm{TM}}$ but further data is required. More botulinum products will be coming onto the market, which will benefit both doctor and consumer in the long term.

The botulinum toxins transformed the cosmetic market with its simple procedure that takes 5-10 minutes and treats almost the whole of the face. There is extensive clinical data, with excellent effects and low incidence of adverse events. For patients it is a short, relatively pain-free, low-cost procedure with high satisfaction rates.

\section{Phosphatidylcholine}

Injecting phosphatidylcholine (PDC) has been shown to reduce small areas of localized or unwanted facial fat. The relative importance of PDC or sodium deoxycholate in producing fat cell lysis is not yet clear. This is still a controversial option and needs more work. The author has used PDC in treating the jowls and chins since 2004 with satisfactory results and low incidence of side effects. ${ }^{40,41}$

\section{Combination therapy}

Unfortunately there is mainly anecdotal evidence for the benefits of combining two or more nonsurgical options. For example it is accepted that when treating deep glabellar folds optimum results are obtained using botulinum toxin to reduce the muscle activity and an HA filler to lift up the fold (Figure 3 ). The toxin can prolong the duration of the filler because mechanical force is part of the degradation process. This clinical benefit was confirmed by Carruthers
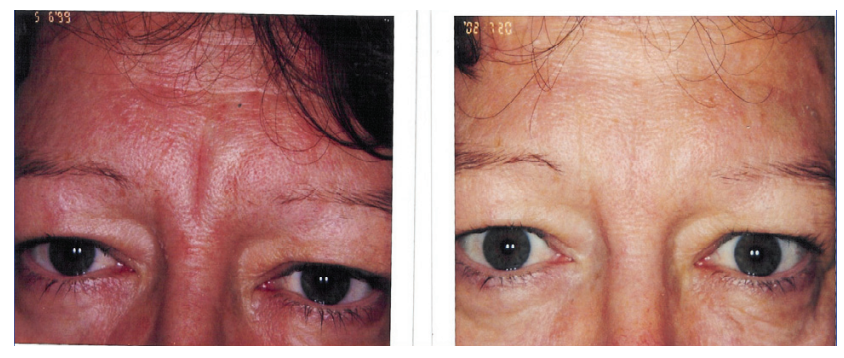

Figure 3 Combination approach: skincare, HA filler, botulinum toxin after three years. 
and Carruthers ${ }^{42}$ who demonstrated an increased duration of effect from 18 to 32 weeks.

Combination of nonsurgical and surgery is becoming increasingly common. Maintaining improvement postsurgery is a logical choice (Figures 3, 4, 5).

\section{Discussion}

The introduction of increasing numbers of nonsurgical options for facial rejuvenation has revolutionized the aesthetic market. Cosmetic treatments were previously the sole province of the rich and famous but in the past 20 years the market has opened up bringing it within reach of a broader spectrum of the population. The public demand for procedures, as demonstrated in the US and UK (Figure 1) is clear. There has been a considerable impact of these changes on the public, medical and allied professions, and governments. In the UK, fuelled by the media, the public seemed to quickly understand and embrace the options. Newspapers, magazines, and radio have regular features and stories on various cosmetic treatments often featuring and endorsed by "celebrities". In the UK, initially doctors were by and large uninterested and the demand was partially satiated by entrepreneurial nurse practitioners, aestheticians, and three large chains of cosmetic surgery clinics. In early 2000s a partial regulation of the UK market took place, governing the use of lasers and IPLs, which for doctors extended to POMs such as botulinum toxin. This government recognition stimulated an interest amongst doctors in a variety of specialties including family doctors, dermatologists, ophthalmic, general and plastic surgeons. Professional bodies, British Association of Plastic Surgery (BAPS) and BAAPS, the British Association of Cosmetic Doctors (BACD), and the British Association of Dermatologists (BAD) began to look at training as they recognized the demand for services. In early 2009 the BACD is launching a diploma in cosmetic medicine through the University of Leicester. This is a first step by UK physicians to bring some organization and credibility to nonsurgical cosmetic medical education.
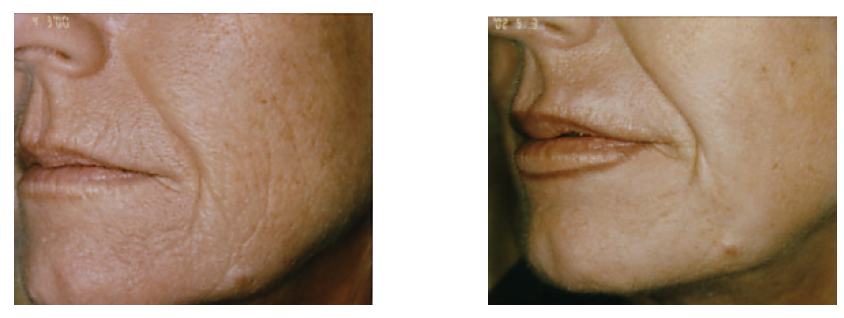

Figure 4 Combination approach:skincare, HA lip enhancement 12 months post-Erbium-Yag laser skin resurfacing.
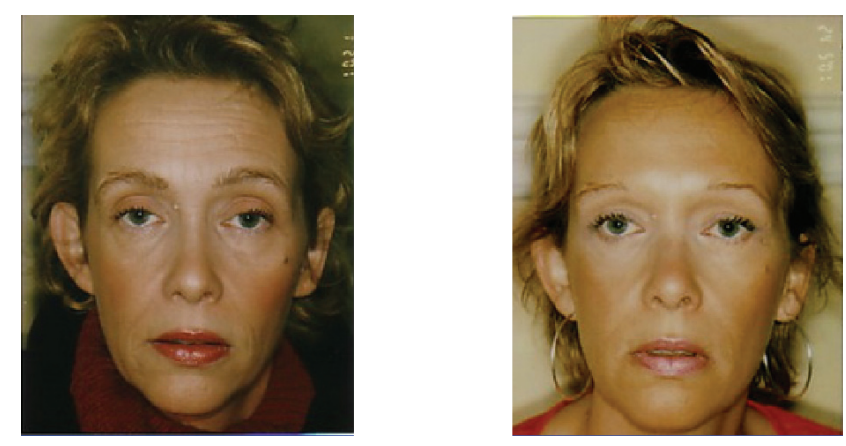

Figure 5 Combination approach: skincare, GA peels, botulinum toxin, HA filler, U\&L blepharoplasty.

\section{Future trends}

In 2008 the UK government proposed a deregulation of the market. It was clear to the majority of interested parties that this was not in the best public interest and following a consultation process the proposal was withdrawn. Other EU member states are about to regulate, so a uniform policy throughout the EU would be desirable. Protection of the public should be a governmental priority and that means ensuring clinics and staff provide high quality, supervised service.

Options for professional, fully accredited cosmetic medical education should become increasingly available as the field becomes recognized as a specialty.

There is an absolute necessity for more clinical studies and data. Botulinum toxin is the only treatment with significant data; all the rest lag behind. The present position that allows suppliers to bring their products to market with minimal study data needs to be reassessed. There are too many commercial pressures demanding early market exposure. However, this has to alter and pressure can be brought by regulators and the medical profession. As new treatment options become available both doctors and public need the security of knowledge and clinical experience that the positives and negatives have been adequately investigated.

This is an exciting field of medicine that is in its infancy and in a constant state of flux. Its growth and development needs to be carefully monitored by the medical profession and government.

\section{Disclosure}

The author reports no conflicts of interest in this work.

\section{References}

1. Mintel report 2007: Cosmetic Surgery - UK - November 2007 [cited 2008 Sept 26]. Available from: http://academic.mintel.com/sinatra/ oxygen_academic/search_results/show\&\&type=RCItem\&sort=a2z\& mode=inaccessible\&list=search_results/display/id=220142.

2. British Association of Aesthetic Plastic Surgeons. BAAPS Audit 2007 [cited 2008 Sept 24]. Available from: http://www.baaps.org.uk. 
3. Kligman AM. Topical retinoic acid for photo aging: conceptions and misperceptions. Cosmet Dermatol. 2003;16(Suppl):S3-S6.

4. Ditre CM. Exfoliants: AHAs and BHAs. In: Draelos ZD, editor. Cosmeceuticals. 1st edition. Philidelphia, PA: Elsevier Saunders; 2005. p. 111-118.

5. Ditre CM, Griffin TD, Murphy GF, et al. Effects of alpha hydroxy acids on photoaged skin: a pilot clinical, histologic and ultrasound study. J Am Acad Dermatol. 1996:34:187-195.

6. Kligman AM. The compatibility of combinations of GA and tretinoin in acne and photoaged facial skin. J Geriatr Dermatol. 1995;3:25A-28A

7. Gilchrest BA. Retinoids and photodamage. Br J Dermatol. 1992; 127(Suppl 41):14-20.

8. Kang S, Leyden JJ, Lowe NJ, et al. Tazarotene cream for treatment of facial photodamage. Arch Dermatol. 2001;1137:1597-1604.

9. Perricone NV. The photoprotective and anti-inflammatory effects of topical ascorbyl palmitate. J Ger Dermatol. 1993;1:5-10.

10. Farris PK. Topical vitamin C: a useful agent for treating photoaging and other dermatologic conditions. Dermatol Surg. 2005;31:814-817.

11. Traikovich SS. Use of topical ascorbic acid and its effects on photodamaged skin topography. Arch Otolaryngol Head Neck Surg. 1999;125:1091-1098.

12. Maaloof S, El-Sabban M, Darwiche N, Gali-Muhtasib H. Protective effect of vitamin $\mathrm{E}$ on UVB light induced damage to in keratinocytes. Mol Carcinog. 2002;34:121-130.

13. McDaniel DH, Neudecker BA, DiNardo JC; Lewis JA, Maibach HI. Idebenone: a new antioxidant of oxidative stress protection capacity compared to commonly known antioxidants $J$ Cosmet Dermatol. 2005;4:10-17.

14. McDaniel D, Neudecker BA, DiNardo JC, Lewis JA, Maibach HI. Clinical efficacy assessment in photodamged skin of $0.5 \%$ and $1.0 \%$ idebeneone. J Cosmet Dermatol. 2005;4:167-173.

15. Balina LM, Graupe K. The treatment of melasma. $20 \%$ azelaic acid versus 4\% hydroquinone. Int J Dermatol. 1991;30:893-895.

16. Brincat MP. Oestrogens and the skin. J Cosmetic Dermatol. 2004;3:41-49.

17. Clark E, Scerri L. Superficial and medium-depth chemical peels. Clin Dermatol. 2008;26:209-218.

18. Landau M. Chemical peels. Clin Dermatol. 2008;26:200-208.

19. Bhalla M, Thami GP. Microdermabrasion: Reappraisal and brief review of literature. Dermatol Surg. 2006;32:809-814.

20. Lee SY, Park KH, Choi JW, et al. A prospective, randomized, placebo controlled, double blinded, split face study on LED phototherapy for skin rejuvenation: Clinical, profilometric, histologic, ultrastructural and biochemical evaluations and comparison of three different treatment settings. J Photochem Photobiol B. 2007;88:51-67.

21. Blyumin ML, Kaufman J. Dermatologic applications of photodynamic therapy. Cosmetic Dermatol. 2008;21:5:290-296.

22. Alexiades-Armenakas MR, Dover JS, Arndt KA. The spectrum of laser skin resurfacing: nonablative, fractional, and ablative laser resurfacing. J Am Acad Dermatol. 2008;58:719-737.

23. Sadick N. Bipolar radiofrequency for facial rejuvenation. Facial Plast Surg Clin North Am. 2007;15:161-167.

24. Preston PW, Lanigan SW. Patient satisfaction with laser hair removal. J Cosmet Dermatol. 2003;2:68-72.

25. Barbucci R, Lamponi S, Magnani A, et al. The influence of molecular weight on the biological activity of heparin like sulfated Hyaluronic acids. Biomaterials. 1998;19:801-806.
26. Nairns RS, Brandt F, Leyden J, et al. A randomised, double-blind multicentre comparison of the efficacy and tolerability of Restylane versus Zyplast for the correction of nasolabial folds. Dermatol Surg. 2003;29:588-595.

27. Wang et al. In vivo stimulation of de novo collagen production caused by cross linked hyaluronic acid dermal filler injections in photodamaged human skin. Arch Dermatol. 2007;143:155-163.

28. Pinsky MA, Thomas JA, Murphy DK, Walker PS. Juvéderm injectable gel: a multicenter, double-blind, randomized study of safety and effectiveness. Aesthetic Surg. 2008;28:17-23.

29. Edwards PC, Fantasia JE. Review of long-term adverse effects associated with the use of chemically-modified animal and non animal source hyaluronic acid dermal fillers. Clin Interv Aging. 2007;2:509-519.

30. Borelli C, Kunte C, Weisenseel P, et al. Deep subcutaneous application of poly lactic acid as a filler for facial lipoatrophy I HIV-infected patients. Skin Pharmacol Physiol. 2005;18:273-288.

31. Sherman RN. Sculptra: the new three dimensional filler. Clini Plast Surg. 2006;33:539-550.

32. Vleggaar D. Poly-L-lactic acid: consultation on the injection techniques. J Eur Acad Dermatol Venereol. 2006;20(Suppl 1):17-21.

33. Carruthers JA, Lowe NJ, Menter MA, et al; BOTOX Glabellar lines Study Group. A multicenter, double-blind, randomized, placebo controlled study of the efficacy and safety of botulinum toxin type A in the treatment of glabellar lines. J Am Acad Dermatol. 2002;46:840-849.

34. Carruthers A, Carruthers J, Cohen J. A prospective, double-blind, randomized, parallel-group, dose-ranging study of botulinum toxin type A in female subjects with horizontal forehead rhytides. Dermatol Surg. 2003;29:461-467.

35. Niamtu III J. Botulinum toxin A: a review of 1,085 oral and maxillofacial patient treatments. J Oral Maxillofac Surg. 2003;61:317-324.

36. Carruthers A, Carruthers J, Lowe NJ, et al; for the BOTOX** Glabellar Lines I and II. One-year, randomised, multicenter, two period study of the safety and efficacy of repeated treatments with botulinum toxin type A in patients with glabellar lines. J Clin Res. 2004;7:1-20.

37. Bowler PJ. A retrospective study in the use of botulinum toxin type A in a UL multidisciplinary cosmetic practice. J Cosmet Dermatol. 2005;4:89-92.

38. Cliff SH, Judodihardjo H, Eltringham E. Different formulations of botulinum toxin type A have different migration characteristics: a double-blind, randomized study. J Cosmet Dermatol. 2008;7:50-54.

39. De Boulle K. 2008. Patient satisfaction with different botulinum toxin type A formulations in the treatment of moderate to severe upper facial rhytids. J Cosmet Laser Ther. 2008;10:87-92.

40. Palmer M, Curran J, Bowler P. Clinical experience and safety in using phosphatidylcholine injections for localised reduction of subcutaneous fat:a multicentre retrospective UK study. J Cosmet Dermatol. 2006;5:218-226.

41. Hasengschwandtner F. Phosphatidylcholine treatment to induce lipolysis. J Cosmet Dermatol. 2005;4:308-313.

42. Carruthers J, Carruthers A. A prospective, randomized, parallel group study analyzing the effect of BTX-A (Botox) and nonanimal sourced hyaluronic acid (NASHA, Restylane) in combination compared with NASHA (Restylane) alone in severe glabellar rhytides in adult female subjects: treatment of severe glabellar rhytides with a hyaluronic acid derivative compared with the derivative and BTX-A. Dermatol Surg. 2003;29:802-809. 
